\title{
Sentinel Lymph Node Biopsy as a Guide for Central Neck Dissection in Patients with Suspicious Thyroid Nodule and Negative Cervical Lymphadenopathy
}

\author{
Mahmoud Saad Farahat, MD; Haitham Mostafa Elmaleh, MD, MRCS \\ Department of General Surgery, Ain Shams University, Cairo, Egypt
}

Background: Routine prophylactic central compartment neck dissection in patients with differentiated thyroid carcinoma is not always accepted as a standard management of occult metastasis due to the risk of hypocalcaemia and recurrent laryngeal nerve injury.

Aim: To evaluate the accuracy of using sentinel lymph node biopsy as a guide in identifying occult cervical LN metastases early and thus to avoid the morbidity associated with routine unnecessary central lymph node dissection.

Patients and methods: A prospective study was conducted in the period between April 2015 and January 2017 in Ain Shams University Hospitals, General Surgery Department, Endocrine Surgery Unit, Cairo, Egypt. The study included fifty two patients who had at least one suspicious thyroid nodule and not accompanied by clinical or radiological cervical $L N$. The patients underwent sentinel lymph node biopsy via intra-tumoral injection of methylene blue dye $1 \%$, followed by tracking the lymphatics till the sentinel LN then total thyroidectomy and central LN dissection.

Results: The study included 52 patients with suspicious thyroid nodule, 11 males and 41 females with mean age $41 \pm 6.7$ (26-65). Co-morbid conditions included DM, HTN and ISHD. Size of nodules ranged from $0.8-5.6 \mathrm{~cm}(1.9 \pm 0.6)$ and most nodules were confined to one lobe $(65.4 \%)$. After the final paraffin pathology 24 nodules were found to be benign and 28 proved malignant. SLNs were identified in 21 of 28

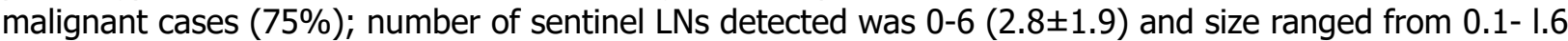
$\mathrm{cm} .18$ of identified SLN were positive for metastasis and 10 were negative while non SLN were positive in $15 / 28$ and negative for $13 / 28$. Thus, the sensitivity, specificity, negative predictive values, positive predictive values and accuracy of SLN biopsy were $85.7 \%, 100 \%, 70 \%, 100 \%$, and $75 \%$, respectively. Post-operative complications included temporary hypocalcaemia in 3 patients and extensive laryngeal edema in 1 patient needing prolonged intubation more than one day then recovered.

Conclusion: SLN biopsy for patients with differentiated thyroid carcinoma and negative LNs clinically and radiologically is an accurate and noninvasive means to identify subclinical lymph node metastasis and so can be used as a guide to determine the need for zone $6 \mathrm{LN}$ dissection.

Key words: Sentinel lymph node, differentiated thyroid carcinoma, zone 6 LN dissection, central neck dissection, STN.

\section{Introduction}

Malignant thyroid tumors are the commonest endocrine tumors, with increasing incidence. ${ }^{1}$ Although thyroid carcinomas have usually excellent prognosis, ${ }^{2}$ presence of cervical lymph node metastasis which reaches about 30\%-90\% of patients with papillary thyroid cancer (PTC) is associated with persistent or recurrent disease. ${ }^{3,4}$ Central compartment (zone 6) is the most common site of metastasis in patients with PTC $^{5}$ and it is proved that central lymph node dissection has been found to decrease local recurrence and improve survival. ${ }^{6}$

Neck dissection is usually associated with significant morbidity including hypo-parathyroidism and injury of the recurrent laryngeal nerve ${ }^{3,7}$ indicating the importance of better selection of patients who would benefit from compartment-oriented lymph node dissection.

The use of the sentinel lymph node (SLN) technique which is defined as the first lymph node in a regional lymphatic area that receives lymph flow from a primary tumor has therefore begun to gain interest as a means to detect occult lymph node metastasis in differentiated thyroid carcinoma (DTC). 8,9

The aim of this study was to determine the efficacy of sentinel lymph node (SLN) biopsy in predicting central compartment metastasis in patients with 
differentiated thyroid carcinoma.

\section{Patients and methods}

The present study was a prospective study conducted in Ain Shams University Hospitals in Egypt, during the period between $1^{\text {st }}$ of April 2015 and 1st of January 2017, and included 52 patients who had at least one suspicious thyroid nodule with clinically and radiologically negative LNs. The patients underwent sentinel lymph node biopsy combined with total thyroidectomy and ipsilateral central $\mathrm{LN}$ dissection. Informed consent was obtained from each patient and all cases were operated on by a consultant surgeon.

Inclusion criteria included any patient with differntiated thyroid carcinoma diagnosed by F.N.A with clinically and radiologically negative LNs regardless age, sex, size of tumor, multifocality or bilaterality. Also patients with highly suspicious nodule for thyroid carcinoma as diagnosed by radiology and FNA were included.

Exclusion criteria included patients not fit for anesthesia, any patient with history of thyroid or neck surgery for non-thyroidal head and neck cancers, palpable or US-detected lymph node involvement and medullary and anaplastic thyroid malignancies. Patients with locally invasive tumors (pT4), initial distant metastases, and patients with history of allergic reactions to drugs were also excluded.

Pre-operative assessment included; full clinical history and clinical examination (general and local), routine pre-operative blood tests (complete blood picture, coagulation profile, liver and kidney functions tests), plain chest $X$-ray, ECG and echocardiography (if indicated), thyroid function test (T.S.H , free T3 \& free T4), neck ultrasound, thyroglobulin level as a baseline,
F.N.A from thyroid nodule and routine vocal cord assessment.

Pre-operative preparation: Control of any coexisting medical disease especially coexisting chest diseases. All patients signed the informed consent and prophylactic dose of antibiotic (amxacillin-claviulenic acid) and dexamethasone were given with induction of anesthesia.

\section{Surgical procedure and detection of SLN:} Under general anesthesia, a standard transverse low-collar skin incision was made; dissection of upper and lower skin flaps was performed and the strap muscles were retracted laterally. Before mobilization, the thyroid gland and the ipsilateral jugular vein were exposed. By using a 30-gauge insulin needle, $0.2-1 \mathrm{~mL}$ of $2 \%$ methylene blue (not more so as not to cause staining of surrounding tissues and this masks lymphatics and becomes misleading) was injected into the tumor (intratumoral); in a large nodule, injection was carried out at 3, 6, 9, 12 o'clock. Within few seconds, the stained lymphatic channels over the thyroid gland were traced to the central compartment and were followed up to the first blue-stained lymph node, which was called the "SLN" and the other LNs in the central compartment were called "non"-SLN. In bilateral nodules the procedure was done on both sides. If there were no stained nodes, the first node closest to the afferent stained lymphatic vessel was considered the SLN. After SLN biopsy, total thyroidectomy with dissection of the ipsilateral central neck compartment and clearance of pretracheal and paratracheal nodes was routinely performed. During the operation the parathyroid glands were preserved on their arterial supply and both recurrent laryngeal nerves were identified and followed to the entrance into the larynx. The SLNs were harvested and sent for paraffin sections.

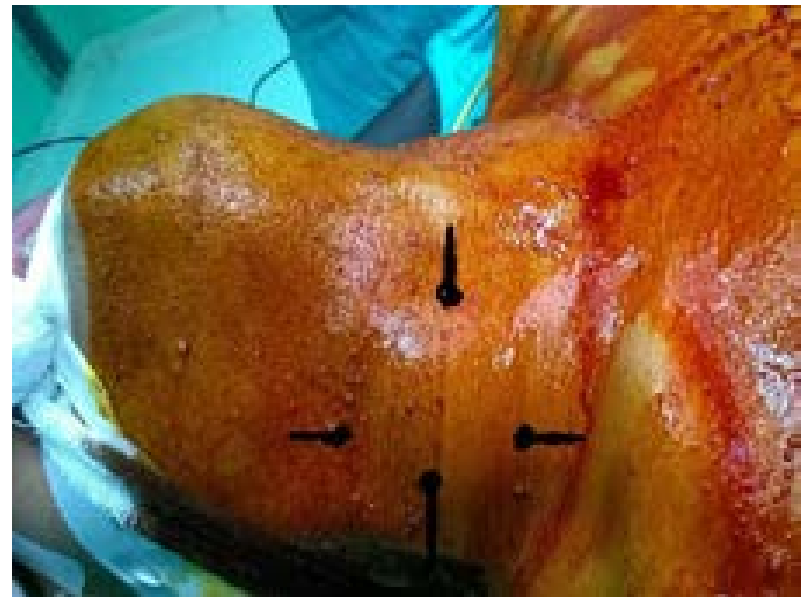

a

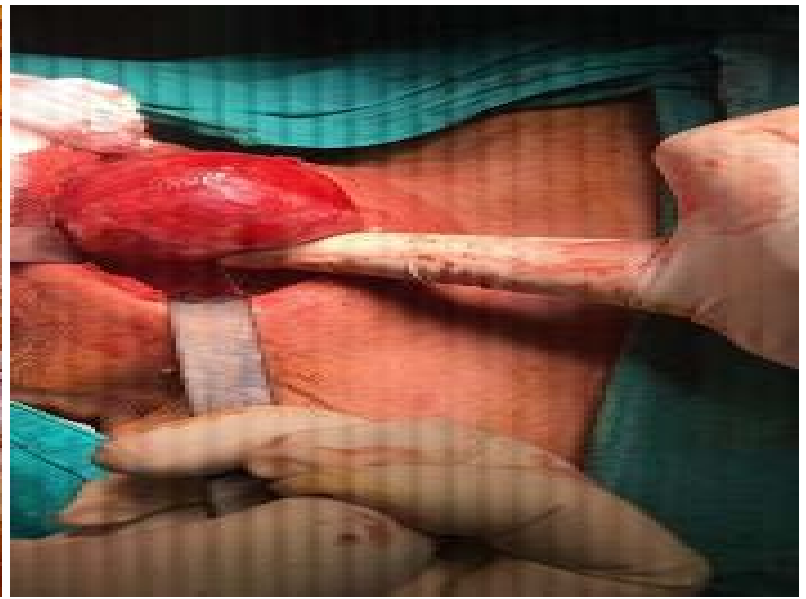

b

Fig 1 (A): Male patient with right thyroid nodule (arrows)

(B): Intra-operative the nodule before dissection of the lobe so as not to cut lymphatics. 


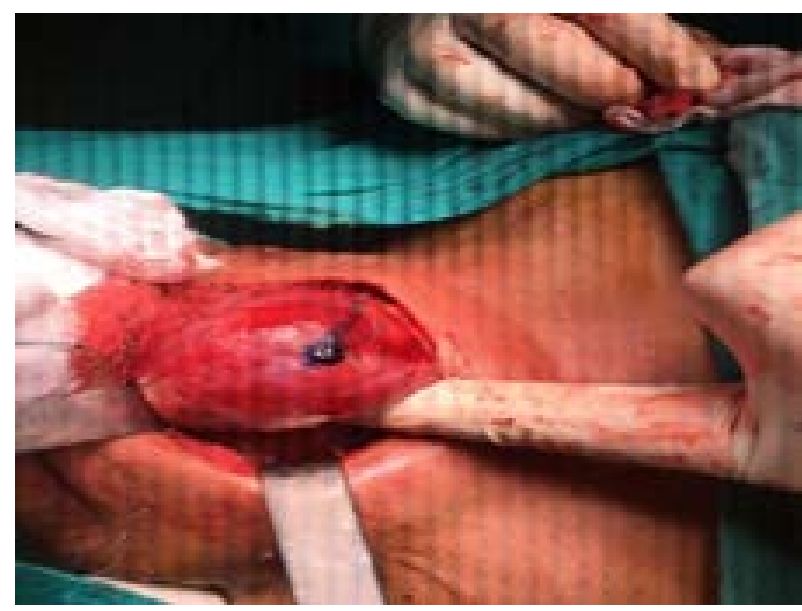

a

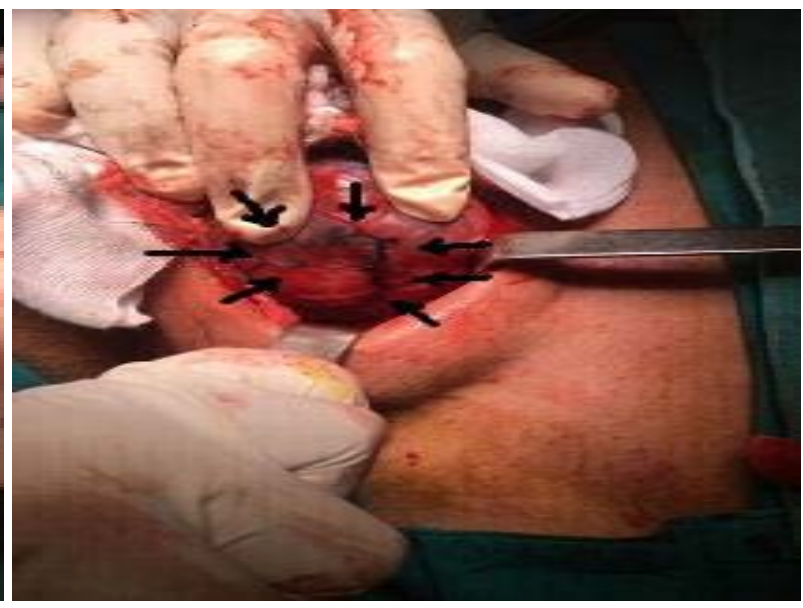

b

Fig 2 (A): Injection of methelyne blue intratumoral (B): Lymphatics arising from the nodule and passing laterally.

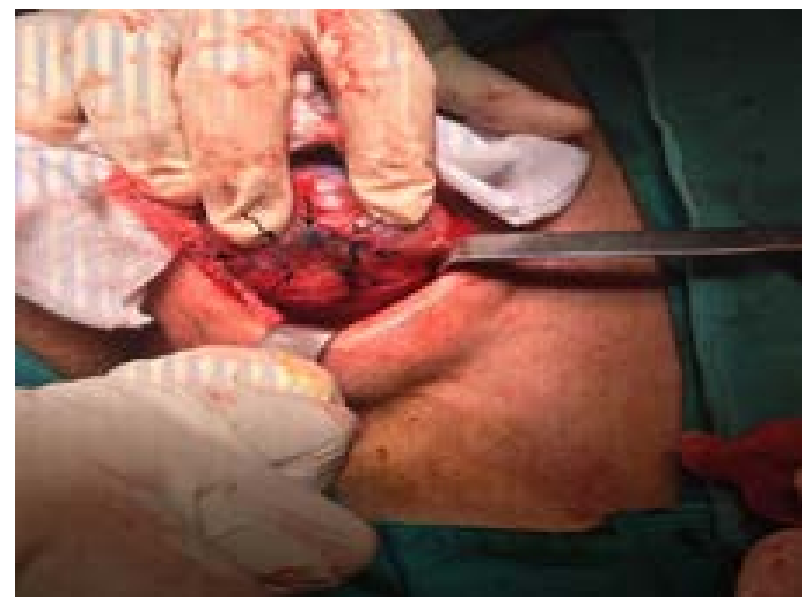

a

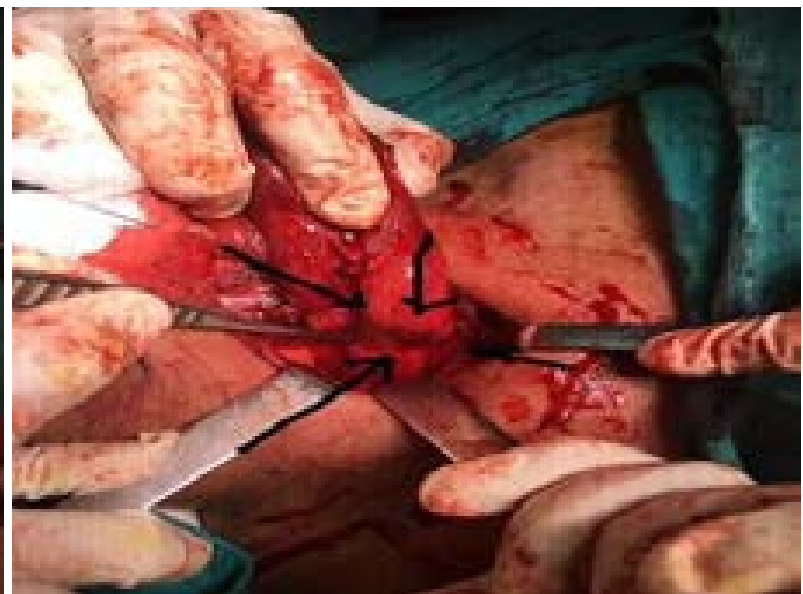

b

Fig 3 (A): Another view of lymphatics passing laterally to the paratracheal LNs (B): Arrows points to the sentinel LNs.

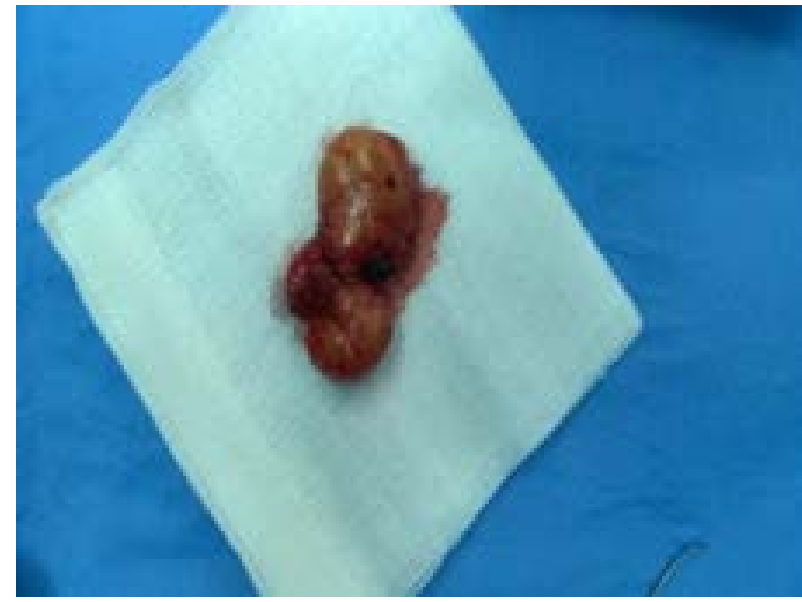

a

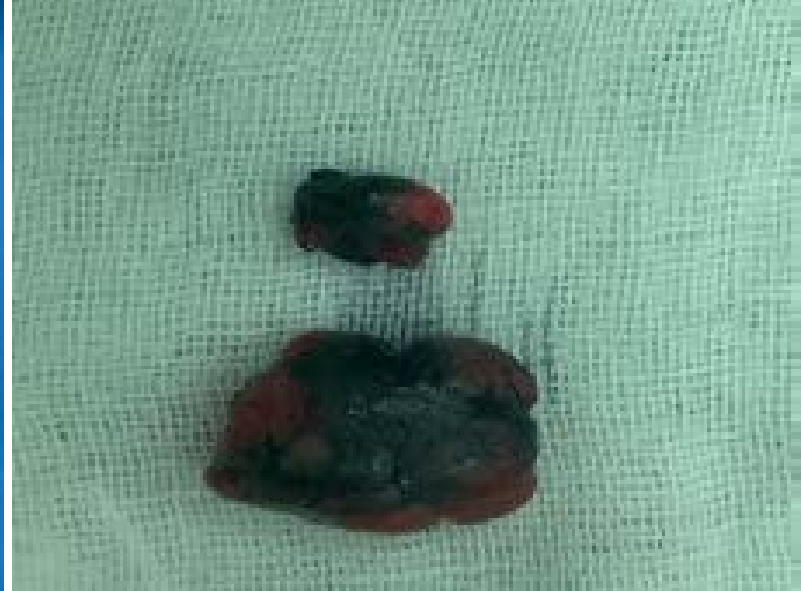

b

Fig 4 (A): (B): LNs after extraction. 
Post-operative follow up: All patients were followed up for any post-operative complications (manifestations of hypocalcemia, voice changes), suppression doses of thyroxine therapy were administered to all patients. All patients were followed regularly by clinical examination and neck US. Neck \& whole-body radioiodine scanning (PET-CT if not available), and serum thyroglobulin measurements were done on regular intervals 1,2 and 3 months.

Statical Analysis: Data were collected tabulated and statically analyzed. Analysis of data was done using SPSS (statistical program for social science version 21) as follows: Descriptive statistics were used to analyze patient and tumor characteristics. Description of quantitative variables was done as number and percentage. Accuracy, sensitivity, specificity, and positive and negative predictive values of the SLN were calculated.

\section{Results}

From April 2015 till January 2017 a prospective study was conducted in Ain Shams University, General Surgery Department, Endocrine Surgery Unit, Cairo, Egypt. Patients admitted in surgery department with suspicious thyroid nodule by F.N.A with clinically and radiologically negative LNs regardless age, sex, size of tumor, multifocality or bilaterality were included in the study. There were 52 patients; 11 males and 41 females with mean age $41 \pm 6.7$ (26-65). Co morbid conditions included DM, HTN, ISHD and morbid obesity.

Table 1: Demographics and associated morbidities

\begin{tabular}{ll}
\hline Age (mean, SD \& range) & $42 \pm 7.5(24-56)$ \\
Sex (no, \%) & $11(21.2 \%)$ \\
Male & $41(78.8 \%)$ \\
Female & \\
Co morbid conditions & 4 \\
DM & 7 \\
HTN & 2 \\
ISHD & \\
\hline
\end{tabular}

Table 2: Pathologic characteristics of the nodules

\begin{tabular}{llll}
\hline Size: & Range $(0.8-5.6)$ mean \pm SD $(1.9 \pm 0.6)$ & No & Percentage \\
\cline { 3 - 4 } & $<1 \mathrm{~cm}(\mathrm{~T} 1)$ & 7 & $13.5 \%$ \\
$1-4 \mathrm{~cm}(\mathrm{~T} 2)$ & 39 & $75 \%$ \\
& $>4 \mathrm{~cm}(\mathrm{~T} 3)$ & 6 & $11.5 \%$ \\
Site: & & & \\
& One lobe & 34 & $65.4 \%$ \\
& Isthmus & 3 & $5.77 \%$ \\
& Bilateral & 15 & $28.85 \%$ \\
\hline
\end{tabular}

Table 3: Final pathology

\begin{tabular}{ll}
\hline $\begin{array}{l}\text { Malignant (28) (53.85\%) } \\
\text { Papillary carcinoma }\end{array}$ & $20(71.43 \%$ of malignant cases $)$ \\
Follicular carcinoma & $3(10.7 \%$ of malignant cases $)$ \\
Hurthle cell carcinoma & $5(17.86 \%$ of malignant cases $)$ \\
Benign (24) (46.15\%) & \\
Follicular adenoma & $11(45.8 \%$ of benign cases $)$ \\
Hurthle cell adenoma & $5(20.8 \%$ of benign cases $)$ \\
Colloid nodule & $8(33.3 \%$ of benign cases $)$ \\
\hline
\end{tabular}


Table 4A: Characteristics of sentinel lymph node (SLN) and non-sentinel lymph node (NSLN)

\begin{tabular}{lcc}
\hline & SLN & Non SLN \\
\cline { 2 - 3 } & No \& Percentage & No \& Percentage \\
\hline Identification rate of SLN & $21 / 28(75 \%)$ & $10 / 28(35.7 \%)$ \\
Number of LN detected (range , mean+ SD) & $0-6\left(2.8+\_1.9\right)$ & $0-9\left(2.4+\_1.3\right)$ \\
Size of SLN detected (range cm) & $0.1-1.6 \mathrm{~cm}$ & $0.1-1.5 \mathrm{~cm}$ \\
LN metastasis found positive & $18 / 28(64.3 \%)$ & $15 / 28(53.6 \%)$ \\
LN metastasis found negative & $10 / 28(35.7 \%)$ & $13 / 28(46.4 \%)$ \\
\hline
\end{tabular}

Table 4B:

\begin{tabular}{lc}
\hline Total number of cases +ve SNL \& NSLN: (total cases with Actual LN disease present) & $21 / 28$ \\
Total number of cases -ve SNL\&NSLN: (total cases actual LN disease absent) & $7 / 28$ \\
Number of cases -ve SNL \& -ve NSLN (true -ve) & 7 \\
Number of cases -ve SNL \& +ve NSLN (false -ve) & 3 \\
Number of cases +ve SNL \& -ve NSLN (false +ve) & 0 \\
\hline
\end{tabular}

\begin{tabular}{lccc}
\hline & Disease present & Disease absen & Total \\
\hline SLN +ve & True +ve (a) & False +ve (b) & $\mathrm{a}+\mathrm{b}$ \\
SLN -ve & False -ve (c) & True -ve (d) & $\mathrm{c}+\mathrm{d}$ \\
Total & $\mathrm{a}+\mathrm{c}$ & $\mathrm{b}+\mathrm{d}$ & $\mathrm{a}+\mathrm{b}+\mathrm{c}+\mathrm{d}$ \\
\hline
\end{tabular}

Sensitivity $=a /(a+c)$, Specificity $=d /(b+d)$, Accuracy $=a+c /(a+b+c+d), \bullet$ Negative predictive value $(N P V)=d /(c+d)$, -Positive predictive value $(P P V)=d /(c+d)$

Table 4: Accuracy of the SLN

\begin{tabular}{lcc}
\hline & No & Percentage \\
\hline Sensitivity & $18 / 21$ & $85.7 \%$ \\
Specificity & $7 / 7$ & $100 \%$ \\
Negative predictive value & $7 / 10$ & $70 \%$ \\
Positive predictive value & $18 / 18$ & $100 \%$ \\
Accuracy & $21 / 28$ & $75 \%$ \\
\hline
\end{tabular}

\section{Discussion}

The prognosis of patients with thyroid carcinoma associated with cervical lymph node metastasis is poor compared to those without LN metastasis. ${ }^{10}$ Factors associated with persistent disease or recurrent papillary thyroid carcinoma are extranodal invasion, lymph node metastasis, a tumor size $>3$ $\mathrm{cm}$, extra-capsular extension, and the locations of metastatic lymph nodes with central compartment lymph nodes being the most frequently involved site. ${ }^{11}$ So, complete surgical removal of lymph nodes in the central compartment during thyroid cancer surgery is important to decrease recurrence and mortality.
Metastasis to LNs in the lateral neck is easily detected clinically and by cervical US but this is not true as regard the metastasis to the central compartment ${ }^{12}$ so subclinical $\mathrm{LN}$ metastasis could be detected by using sentinel lymph nodes (SLN). Sentinel lymph node is defined as the first lymph node draining a regional lymphatic from a primary tumor. ${ }^{13,14}$ SLN can avoid unnecessary lymph node dissection in patients with negative LNs (cNO).

The idea of sentinel lymph nodes (SLN) as the first node(s) to drain from a tumor was described by Ramon Cabanas ${ }^{15}$ and later on was established in skin melanoma ${ }^{16}$ and breast cancer. ${ }^{17,18}$ Controversies in the surgical management of 
lymph nodes in DTC, have led some authors to propose that SLN should be sampled in patients with thyroid cancer to support the decision whether to perform lymph node dissection or not.

The aim of our study was to assess the use of SLN biopsy in detecting early metastasis to the central lymph node compartment in patients with differentiated thyroid carcinoma without clinically or radiologically detected LNs (cNO).

Our study was conducted on 52 patients who had at least one suspicious thyroid nodule, the size of nodules ranged from $0.8-5.6 \mathrm{~cm}$ with a mean of $1.9 \pm 0.6$. Sizes of most nodules ranged between one and four $\mathrm{cm}(75 \%)$ and were present mostly in one lobe (65.4\%) and to lesser extent bilaterally, and the isthmus was the least (5.77\%). Final paraffin pathological reports showed that 24 patients $(46.15 \%)$ had benign nodule with the follicular adenoma as the most frequent. Malignant nodules were present in 28 patients $(53.85 \%)$ and with the papillary thyroid cancer as the most frequent cancer $(71.43 \%)$.

In our patients, we used methylene blue injected inside the thyroid nodule (intra-tumoral) to identify the sentinel LN. Other methods for identifying SLN in differentiated thyroid carcinoma include intraoperative gamma probe and lymphoscintigraphy. ${ }^{9}$ Both are accurate methods but are expensive, time consuming and not available, also hypersensitivity and anaphylaxis have been reported $1-3 \%$ when isosulfane blue dye was used. ${ }^{19}$ Methylene blue has the advantage of being available, cheap and there is no significant difference between its results and isosulfane in identifying SLN. ${ }^{20}$

In our study, 3 patients developed transient hypocalcemia that were treated with $\mathrm{Ca}$ and Onealfa and all improved in 3-4 weeks post-operative. No cases developed recurrent laryngeal nerve injury.

In our patients, sentinel LNs were identified in $21 / 28$ patients of malignant cases (accuracy). Number of LNs ranged from 0-6 (12 \pm 1.9$)$ and size ranged from 0.1-1.6 cm, 18/28 (64.3\%). SLN were positive for metastasis and 10/28 (35.7\%) were negative for metastasis. In $10 / 24$ patients (41.7\%) of benign nodules, lymph nodes were found and all were reactive, non sentinel LNs were extracted via the central compartment dissection. 15/28 were found positive for metastasis and $13 / 28$ were found negative.

From our results SLN had sensitivity, specificity, negative predictive value, positive predictive value and accuracy of $85.7 \%, 100 \%, 70 \%, 100 \%, 75 \%$ respectively.
Kelemen et al. In $1998^{21}$ had injected isosulfan blue dye inside suspicious nodules followed by total thyroidectomy in all patients except one (lobectomy). Kelemen found 12/17 malignant cases and $5 / 17$ benign (follicular adenoma \& colloid nodules) cases. Size ranged from $0.8-4 \mathrm{~cm}$ but he didn't find any lymph nodes in benign cases. In 2003, Takami et al. ${ }^{22}$ injected Isosulfane blue dye around the tumor, all their patients were diagnosed as papillary thyroid cancer pre-operatively by FNA and all underwent subtotal thyroidectomy and central and modified neck dissection. Dzodic in $2006^{23}$ carried on his work on differentiated thyroid cancer by peritumoral injection of methylene blue and SLNs were traced in the lower third of jugulo carotid chain and patients underwent total thyroidectomy and routine central neck dissection, Roh and park, $2008^{8}$ injected methylene blue peritumoral in newely diagnosed papillary thyroid cancer followed by total thyroidectomy and central neck dissection. Anand et al., $2009^{24}$ studied SLN in suspicious nodule by injecting methylene blue peri-tumoral followed by total thyroidectomy and central compartment lymph node dissection.

Takami et al in $2003^{22}$ found that SLN have a sensitivity of $87.55 \%$ (35/40), specificity of $100 \%$ (23/23), positive predictive value of $100 \%$ (35/35), negative predictive value of $82.1 \%(23-28)$ and accuracy of $92.1 \%$ (58/63). Dzokc in $2006^{23}$ found the sensitivity of SLN to be $77.7 \%$, specificity $100 \%$, negative predictive value $94 \%$, positive predictive value $100 \%$ and overall accuracy $95 \%$.

Anand et al in $2009^{24}$ reported an accuracy, sensitivity, specificity, positive predictive value and negative predictive value of SLN as $87 \%, 100 \%$, $86 \%, 40 \%$ and $100 \%$ respectively. While Roh and Park in $2008^{8}$ found the sensitivity, specificity, accuracy, positive and negative predictive values to be $77.8 \%, 100 \%, 92 \%, 100 \%$ and $88.9 \%$ respectively.

\section{Conclusion}

From our study we concluded that in patients having differentiated carcinoma of thyroid without evidence of macroscopic clinical or US-detected lymph node metastasis, sentinel lymph node biopsy can discover occult metastases with high accuracy and no morbidity. Improvement of lymph node metastatic identification using SLN biopsy may help select patients who would benefit from central compartment neck dissection and sparing those who do not need.

\section{References}

1. Jung KW, Park S, Kong HJ, Won YJ, Lee JY, Seo HG, et al.: Cancer statistics in Korea: incidence, mortality, survival, and prevalence in 2009. Cancer Res Treat 2012; 44: 11-24. 
2. Ahmadi N, Grewal A, Davidson BJ: Patterns of cervical lymph node metastases in primary and recurrent papillary thyroid cancer. $\boldsymbol{J}$ Oncol 2011; 2011: 735-678.

3. Roh JL, Park JY, Park CI: Total thyroidectomy plus neck dissection in differentiated papillary thyroid carcinoma patients: Pattern of nodal metastasis, morbidity, recurrence, and postoperative levels of serum parathyroid hormone. Ann Surg 2007; 245: 604-610.

4. Zhang L, Wei WJ, Ji QH, Zhu YX, Wang ZY, Wang $Y$, et al.: Risk factors for neck nodal metastasis in papillary thyroid microcarcinoma: A study of 1066 patients. J Clin Endocrinol Metab 2012; 97: 1250-1257.

5. American Thyroid Association Surgery Working Group: American Association of Endocrine Surgeons; American Academy of Otolaryngology-Head and Neck Surgery; American Head and Neck Society. Carty SE, Cooper DS, et al. Consensus statement on the terminology and classification of central neck dissection for thyroid cancer. Thyroid 2009; 19: $1153-1158$.

6. Wang TS, Evans DB, Fareau GG, Carroll T, Yen TW: Effect of prophylactic central compartment neck dissection on serum thyroglobulin and recommendations for adjuvant radioactive iodine in patients with differentiated thyroid cancer. Ann Surg Oncol 2012; 19: 4217-4222.

7. Pereira $J A$, Jimeno $J$, Miquel $J$, Iglesias $M$, Munné A, Sancho JJ, et al.: Nodal yield, morbidity, and recurrence after central neck dissection for papillary thyroid carcinoma. Surgery 2005; 138: 1095-1100.

8. Roh JL, Park CI: Sentinel lymph node biopsy as guidance for central neck dissection in patients with papillary thyroid carcinoma. Cancer 2008; 113: 1527- 1531.

9. Carcoforo P, Feggi L, Trasforini G, Lanzara S., Sortini D, Zulian V., et al.: Use of preoperative lymphoscintigraphy and intraoperative gamma-probe detection for identification of the sentinel lymph node in patients with papillary thyroid carcinoma. Eur J Surg Oncol 2007; 33: 1075- 1080.

10. Lundgren CI, Hall P, Dickman PW, Zedenius J: Clinically significant prognostic factors for differentiated thyroid carcinoma: A populationbased, nested case-control study. Cancer 2006; 106: 524-531.
11. Gemsenjäger E, Perren A, Seifert B, Schüler G, Schweizer I, Heitz PU: Lymph node surgery in papillary thyroid carcinoma. $\boldsymbol{J}$ Am Coll Surg 2003; 197: 182-190.

12. Rosario PW, de Faria S, Bicalho L, Alves MF, Borges MA, Purisch $S$, et al.: Ultrasonographic differentiation between metastatic and benign lymph nodes in patients with papillary thyroid carcinoma. J Ultrasound Med 2005; 24: 13851389.

13. Pelizzo MR, Rubello $D$, Boschin IM, Piotto $A$, Paggetta C, Toniato A, et al.: Contribution of SLN investigation with 99mTc-nanocolloid in clinical staging of thyroid cancer: Technical feasibility. Eur J Nucl Med Mol Imaging 2007; 34: 934- 938.

14. Chen SL, Iddings DM, Scheri RP, Bilchik AJ: Lymphatic mapping and sentinel node analysis: Current concepts and applications. CA Cancer J Clin 2006; 56: 292- 309.

15. Cabanas RM: An approach for the treatment of penile carcinoma. Cancer 1977; 39: 456-466.

16. Morton DL, Wen D-R, Wong JH, Economou JS, Cagle LA, Storm FK, et al.: Technical details of intraoperative lymphatic mapping for early stage melanoma. Arch Surg 1992; 127: 392399.

17. Giuliano $A E$, Kirgan $D M$, Guenther $J M$, Morton DL: Lymphatic mapping and sentinel lymphadenectomy for breast cancer. Ann Surg 1994; 220: 391-401.

18. Krag DN, Weaver DL, Alex JC, Fairbank JT: Surgical resection and radio localisation of the sentinel node in breast cancer using gamma probe. Surg Oncol 1993; 2: 335-340.

19. Montgomery LL, Thorne AC, Van Zee KJ, Fey J, Heerdt AS, Gemignani $M$, et al.: Isosulfan blue dye reactions during sentinel lymph node mapping for breast cancer. Anesth Analg 2002; 95: 385- 388.

20. Thevarajah S, Huston TL, Simmons RM: A comparison of the adverse reactions associated with isosulfan blue versus methylene blue dye in sentinel lymph node biopsy for breast cancer. Am J Surg 2005; 189: 236- 239.

21. Keleman PR, Van Herle AJ, Giuliano AE: Sentinel lymphadenectomy in thyroid malignant neoplasms, Arch Surg 1998; 133: 288-292. 
22. Takami H, Sasaki K, Ikeda $Y$, Tajima G, Kameyama K: Detection of sentinel lymph nodes in patients with papillary thyroid cancer. Asian Journal of Surgery 2003; 26: 145-8.

23. Dzodic R: Sentinel lymph node biopsy may be used to support the decision to perform modified radical neck dissection in differentiated thyroid carcinoma World J Surg 2006; 30: 841-846.

24. Anand S M., Gologan O, Rochon L, Tamilia M, How J., Hier MP, et al.: The role of sentinel lymph node biopsy in differentiated thyroid carcinoma. Arch Otolaryngol Head Neck Surg 2009; 135: 1199-1204. 